\title{
The chemokine KC, but not monocyte chemoattractant protein-1, triggers monocyte arrest on early atherosclerotic endothelium
}

\author{
Yuqing Huo, ${ }^{1}$ Christian Weber, ${ }^{2}$ S.B. Forlow, ${ }^{1}$ Markus Sperandio, ${ }^{1}$ Jayant Thatte, ${ }^{1}$ \\ Matthias Mack, ${ }^{3}$ Steffen Jung, ${ }^{4}$ Dan R. Littman, ${ }^{4}$ and Klaus Ley ${ }^{1}$ \\ ${ }^{1}$ Cardiovascular Research Center and Department of Biomedical Engineering, University of Virginia, Health Science Center, \\ Charlottesville, Virginia, USA \\ ${ }^{2}$ Department of Molecular Cardiovascular Research, University Hospital Aachen, Aachen, Germany \\ ${ }^{3}$ Medical Policlinic, University of Munich, Munich, Germany \\ ${ }^{4}$ Skirball Institute of Biomolecular Medicine and Howard Hughes Medical Institute, New York, New York, USA
}

Address correspondence to: Klaus Ley, Department of Biomedical Engineering, University of Virginia Health Science Center, Box 800759, Charlottesville, Virginia 22908, USA. Phone: (434) 924-1722; Fax: (434) 982-3870; E-mail: klausley@virginia.edu.

Received for publication April 2, 2001, and accepted in revised form September 10, 2001.

In a reconstituted flow chamber system, preincubation with chemokines can trigger the arrest of rolling monocytes, suggesting that this interaction could help recruit these cells to early atherosclerotic lesions. To date, however, the contribution of endothelium-derived chemokines found in these lesion to monocyte arrests has not been investigated. The endothelium of lesion-prone carotid arteries from apolipoprotein E-deficient $\left(\mathrm{ApoE}^{-/-}\right)$mice, but not control mice, presents the chemokines KC (mouse GRO- $\alpha$ ) and JE (mouse monocyte chemoattractant protein-1 [MCP-1]). Arrest of a monocytic cell line or mouse blood monocytes perfused through carotid arteries of $A \mathrm{POE}^{-/-}$mice was reduced by treating with either pertussis toxin, an antagonist of CXCR2, or an antibody to KC, but this process was insensitive to agents that blocked CCR-2 or JE. Conversely, monocyte accumulation more than doubled upon pre-perfusion of the carotid artery with KC but not with mouse MCP-1. Blockade of $\alpha_{4} \beta_{1}$ integrin (VLA-4) or vascular cell adhesion molecule-1, but not CD18 or intercellular adhesion molecule-1, almost completely inhibited the arrest of monocytes. We conclude that when presented by early atherosclerotic lesions, KC but not murine MCP-1 triggers VLA-4-dependent monocyte recruitment.

J. Clin. Invest. 108:1307-1314 (2001). DOI:10.1172/JCI200112877.

\section{Introduction}

Atherosclerosis is an inflammatory disease of the artery wall (1). Atherogenesis requires a complex interplay between mononuclear cells, endothelial cells, vascular smooth muscle cells, growth factors, and cytokines (2). The formation of atherosclerotic lesions proceeds through a sequence from fatty streak to fibrofatty matrix and fibrous plaque. Monocyte arrest on vascular endothelial lining is not only considered to be an initial step, but appears to play a causative role in the ensuing pathological process $(3,4)$.

The mechanisms by which monocytes arrest on the luminal surface of vessels prone to form atherosclerotic lesions are incompletely understood. The adhesion molecules P-selectin, intercellular adhesion molecule1 (ICAM-1) (5) and vascular cell adhesion molecule-1 (VCAM-1) (6) have been detected on the luminal surface of vessels with nascent or established lesions. In animal models, endothelial areas expressing P-selectin and VCAM-1, but not ICAM-1, have been shown to be closely correlated with monocyte/macrophage infiltration and lesion formation $(7,8)$.

Apolipoprotein E deficient (apoE $\mathrm{E}^{-/}$) mice develop spontaneous atherosclerotic lesions in the arterial vasculature with a pattern similar to that observed in humans (9). In the ex vivo perfused carotid artery of the apoE $-/-$ mouse, we have recently shown that P-selectin and its ligand PSGL-1, and VCAM- 1 and its ligand VLA-4, are critical for monocyte accumulation on the luminal surface of the carotid bifurcation (10, 11), a predilection site for atherosclerotic lesions. Formation of lesions was markedly reduced in atherosclerosis-prone mice deficient in P-selectin $(12,13)$ or after peptide perfusion to block VLA-4 (14) compared with control mice.

Chemokines are a superfamily of structurally related small chemotactic cytokines involved in leukocyte trafficking and activation. Chemokines signal through seven transmembrane receptors linked to the pertussis toxin-sensitive (PTX-sensitive) $\alpha$ subunit of $G_{i}$ $\left(\mathrm{G}_{\mathrm{i}} \alpha\right)$ to cause activation of phospholipase $\mathrm{C}$ and PI3 kinase (15). Binding of chemokines to their receptors elicits a variety of cellular responses including an increase in intracellular free calcium concentration, integrin activation, and leukocyte migration (16). Chemokines can trigger rapid activation of integrindependent leukocyte arrest on endothelium or other substrates (17). Monocyte chemoattractant protein 
(MCP-1) (18), IL-8 (19), RANTES (20), macrophage inflammatory protein-1 (MIP-1) (21), and other chemokines have been detected in atherosclerotic lesions. Atherosclerosis-prone mice lacking MCP-1 (22) or its receptor CCR2 (23) have a reduced ability to recruit monocytes to atherosclerotic lesion sites and develop fewer and smaller atherosclerotic lesions than do control apoE ${ }^{-/}$-mice. To further investigate the role of chemokines in recruitment of monocytes into the vessel wall, monocyte arrest has been studied on endothelial cells in vitro. Under flow conditions, monocytes preincubated with MCP- 1 or IL- 8 adhere on endothelial cells infected with an adenovirus encoding E-selectin (24). Arrest of monocytes to cultured endothelial cells in shear flow was also found to be promoted by surface-bond GRO- $\alpha$ (25).

On the basis of these studies, we hypothesized that arrest chemokines present on the endothelium of lesion-prone sites in arteries may trigger monocyte arrest. Such a chemokine would be defined as a relevant arrest chemokine if (a) it is expressed on atherosclerotic endothelium, (b) its blockade reduces monocytes arrest, and (c) its addition increases monocyte arrest. Here we show that $\mathrm{KC}$, the murine homologue of GRO- $\alpha$ (26) but not JE, the murine homologue of MCP-1 (27) triggers arrest of monocytes in early atherosclerotic carotid arteries via VLA-4 and VCAM-1. To assess the involvement of chemokine-mediated activation in monocyte arrest, PTX, which blocks $\mathrm{G}_{\mathrm{i}}$-mediated signaling by ADP ribosylation (28), and mutant PTX lacking ADP-ribosyltransferase activity (29), were used. Peptides and antibodies blocking chemokines or their receptors were used to identify chemokines that are important for monocyte arrest on early atherosclerotic lesions.

\section{Methods}

$m A b$ 's and peptides. Rat mAb's to mouse VLA-4 (PS/2; IgG2b; American Type Culture Collection, Manassas, Virginia, USA), VCAM-1 (MK/2.7; IgG1; ATCC), and ICAM-1 (YN1; IgG2b; ATCC), and mouse mAb's to human CD18 (IB4; IgG2 $\alpha$; ATCC) were purified from hybridoma supernatants. Mouse anti-human VLA-4 integrin (HP2/1, IgG1) was purchased from Immunotech (Westbrook, Maine, USA). Rat antimouse CD18 (GAME-46, IgG1) and control rat IgG1 and IgG2b and mouse IgG1 were purchased from PharMingen (San Diego, California, USA). Polyclonal goat anti-mouse KC, JE, MIP-2, and goat IgG were purchased from R \& D Systems Inc. (Minneapolis, Minnesota, USA). Polyclonal goat anti-mouse MCP-1 was purchased from Santa Cruz Biotechnology (Santa Cruz, California, USA). Mouse MCP-1, MIP-2, and KC were purchased from PeproTech Inc. (Rocky Hill, New Jersey, USA). The 8-73 GRO- $\alpha$ and 9-76 MCP-1 peptide analogues were synthesized as described previously (30-32). PTX and mutant PTX (pertussis holotoxin, PT9K 129G) were provided by E. Hewlett (University of Virginia, Charlottesville, Virginia, USA).
Monocytic cells and monocytes. The monocytic cell line Mono Mac 6 (MM6) (33) was cultured in 24-well plates in RPMI 1640 supplemented with 20\% FCS (Atlanta Biological Inc., Norcross, Georgia, USA), L-glutamine (2 $\mathrm{mM})$, penicillin $(200 \mathrm{U} / \mathrm{ml})$, streptomycin $(200$ $\mu \mathrm{g} / \mathrm{ml}$; Life Technologies Inc., Grand Island, New York, USA), nonessential amino acids (Life Technologies Inc.) and OPI-supplement containing oxalacetic acid, sodium pyruvate and insulin (Sigma Chemical Co., St. Louis, Missouri, USA).

Given that mouse blood monocytes cannot be isolated, we used mononuclear cells from C57BL/6 $\mathrm{CX}_{3} \mathrm{CR} 1^{\mathrm{GFP} /+}$ mice (34). In these mice, a green fluorescent protein (GFP) reporter gene replaced one allele of the CX3CR1 gene, making all murine monocytes and subsets of NK cells, but not other leukocytes, visible under stroboscopic epifluorescence microscopy (34). Because we used mononuclear cell preparations, we cannot formally exclude that the arrest of lymphocytes (not fluorescent) might have secondary effect on monocyte arrest. To produce enough GFP-expressing monocytes, bone marrow from $\mathrm{C} 57 \mathrm{BL} / 6 \mathrm{CX}_{3} \mathrm{CR} 1^{\mathrm{GFP} /+}$ mice was harvested and injected into lethally irradiated C57BL/ 6 mice as described elsewhere (35). Bone marrow cells from one $\mathrm{C} 57 \mathrm{BL} / 6 \mathrm{CX}_{3} \mathrm{CR} 1^{\mathrm{GFP} /+}$ can reconstitute as many as 10 to $20 \mathrm{C} 57 \mathrm{BL} / 6$ mice. Four weeks after bone marrow transplant (BMT), blood was drawn from four to eight mice via carotid artery catheters. Murine blood $(1 \mathrm{ml})$ was layered over $5 \mathrm{ml}$ of animal mononuclear separation solution 1.077 (Accurate Chemical \& Scientific Corp., Westbury, New York, USA) and centrifuged at $600 \mathrm{~g}$ for 20 minutes. Mononuclear cells were collected in $10 \mathrm{ml}$ of $0.9 \% \mathrm{NaCl}$ with $0.13 \%$ (wt/vol) EDTA and $1 \%$ human serum albumin (HSA). To remove platelets, the suspension was centrifuged at $300 \mathrm{~g}$ for 10 minutes and resuspended in Hanks' solution without $\mathrm{Ca}^{2+}$ and $\mathrm{Mg}^{2+}$ with $0.5 \mathrm{mg} / \mathrm{ml} \mathrm{HSA}$ and $20 \mathrm{mM}$ of HEPES. This procedure yields pure (>97\%) mononuclear cells. More than $75 \%$ of the GFP-expressing mouse leukocytes perfused through the carotid artery and visible under epifluorescence microscopy are primary monocytes (34).

MM6 cells or mouse monocytes were incubated with PTX or mutant PTX at $250 \mathrm{ng} / \mathrm{ml}$ for 2 hours. Either 9-76MCP- 1 or $8-73 \mathrm{GRO}-\alpha$ was used at $5 \mu \mathrm{g} / \mathrm{ml}$ for 20 minutes at room temperature, based on the observation that $9-76 \mathrm{MCP}-1$ at $5 \mu \mathrm{g} / \mathrm{ml}$ inhibited arthritis in the MRL-lpr mouse model (36). The cell suspension was warmed to $37^{\circ} \mathrm{C}$, and $\mathrm{Ca}^{2+}$ and $\mathrm{Mg}^{2+}$ were added to a final concentration of $1 \mathrm{mM}$, immediately loaded into $3-\mathrm{cm}^{3}$ syringe, and perfused into the carotid artery using a continuous syringe pump.

Ex vivo perfusion of murine carotid arteries. Male homozygous apoE-/- mice on the C57BL/6J background were from The Jackson Laboratory (Bar Harbor, Maine, USA). At 6 weeks of age, mice were placed on a Western-type diet $(21 \%$ fat; $0.15 \%$ cholesterol wt/wt [Teklad Adjusted Calories Diet TD 88137; Harlan Teklad Laboratory, Madison, Wisconsin, USA]) for $4-5$ weeks. As described previously $(10,11)$, one carotid 
artery per mouse was cannulated and perfused with MOPS-buffered physiological salt solution supplemented with $1 \%$ JBSA and hirudin $(0.5 \mathrm{U} / \mathrm{ml})$ via a syringe pump. MM6 $\left(1 \times 10^{6} / \mathrm{ml}\right)$ labeled with calcein AM (Molecular Probes Inc., Eugene, Oregon, USA) or murine mononuclear cell isolated from $\mathrm{C} 57 \mathrm{BL} / 6$ $\mathrm{CX}_{3} \mathrm{CR} 1^{\mathrm{GFP} /+}$ (monocyte concentration: $4 \times 10^{5} / \mathrm{ml}$ ) were perfused at $5-10 \mu \mathrm{l} / \mathrm{min}$ (resulting in about $\left.1.5-3.0 \mathrm{dyn} / \mathrm{cm}^{2}\right)(10)$. Cell rolling and arrest on endothelium of bifurcation of carotid arteries were recorded on videotape by means of stroboscopic epifluorescence illumination with an intravital microscope (Axioskop, Carl Zeiss Inc., Thornwood, New York, USA) $20 \times$ water immersion objective; 0.5 numerical aperture. Some carotid arteries were perfused with blocking antibody to chemokines for 20 minutes at 50 $\mu \mathrm{g} / \mathrm{ml}$ or preperfused with chemokines at $100 \mathrm{nM}$ for 5 minutes, and then rinsed with MOPS solution.

Immunohistochemistry. Immunostaining for KC, MCP-1, and MIP- 2 was performed on $5-\mu \mathrm{m}$ paraffin sections of murine carotid arteries. Slides were incubated with avidin-biotin blocking reagent containing 10\% horse serum (Vector Laboratories, Burlingame, California, USA) followed by primary antibody $(5 \mu \mathrm{g} / \mathrm{ml}$ of polyclonal goat anti-mouse $\mathrm{KC} ; 5 \mu \mathrm{g} / \mathrm{ml}$ of anti-MIP-2 [R\& D Systems Inc.] or $1 \mu \mathrm{g} / \mathrm{ml}$ of polyclonal anti-mouse MCP-1 [Santa Cruz Biotechnology]) or goat IgG in the presence of $10 \%$ horse serum overnight at $4^{\circ} \mathrm{C}$ followed by biotin-conjugated horse anti-goat IgG, avidin-biotin complex, and 3,3'-diaminobenzidine as substrate (Vector Laboratories). Slides were counterstained with hematoxylin, dehydrated, and examined under a light microscope (Zeiss $100 \times / 1.4$ oil immersion objective).

Transmigration assay. Transmigration assays were performed as described previously (37) using uncoated 6.5 - $\mathrm{mm}$-diameter Transwell inserts $(5-\mu \mathrm{m}$ pore size). JE in assay medium (RPMI 1640/medium 199 and 0.5\% HSA) was added to 24-well tissue culture plates at the final concentrations indicated in Figure 3b. Transwells were inserted, and cells were added to the top chamber. A dilution of cells served as a measure of input. For inhibition studies, JE blocking antibody or CCR2 antagonist 9-76MCP-1 was added. MM6 cells were allowed to transmigrate for 3 hours. Input and transmigrated cells were counted by flow cytometry with appropriate light scatter settings using standard beads. Statistical analysis. Data are represented as the mean $\pm \mathrm{SE}$ of at least four independent experiments and were compared using two-tailed Student's $t$ test. The null hypothesis was rejected at $P<0.05$.

\section{Results}

Monocyte activation through $G_{i} \alpha$-coupled receptors contributes to their arrest on native early atherosclerotic endothelium. MM6 cells perfused through carotid arteries of apoE-/mice accumulated on the endothelial surface at a rate of about six cells per minute during the first 5 minutes of perfusion. Almost all monocytes rolled for a short distance before becoming adherent. After incubation with
PTX, MM6 cells were still able to roll on the endothelium of the bifurcation area at the normal rate (data not shown), but their accumulation was significantly reduced by more than $60 \%$ compared with untreated MM6 cells or with MM6 cells treated with inactive mutant PTX $(n=9 ; P<0.01$; Figure 1a). Accumulation of mouse monocytes treated with PTX was reduced to a similar extent $(n=4 ; P<0.01$; Figure $1 \mathrm{~b})$.

Chemokines are presented on native early atherosclerotic endothelium. To determine whether relevant chemokines are expressed on early atherosclerotic endothelium, we performed immunohistology on carotid artery sections from apoE $\mathrm{E}^{-/-}$mice fed with a Western diet for 5 weeks. KC and MCP-1 were both detected (Figure 2, b and c). No staining was seen on the same vessel using goat $\operatorname{Ig} G$ as the first antibody (Figure 2a). Staining for KC and MCP-1 also diffusely distributed throughout the vessel wall but not in periadventitial tissue.

KC present on early atherosclerotic endothelium is involved in monocyte arrest in situ. To investigate whether chemokines and their receptors were involved in monocyte arrest on early atherosclerotic endothelium, we used receptor antagonists and antibodies to chemokines. MM6 cells express the relevant chemokine receptors CCR2 for MCP-1 and CXCR2 for GRO- $\alpha$ at a level similar to that of primary human monocytes (25). MM6 cells showed
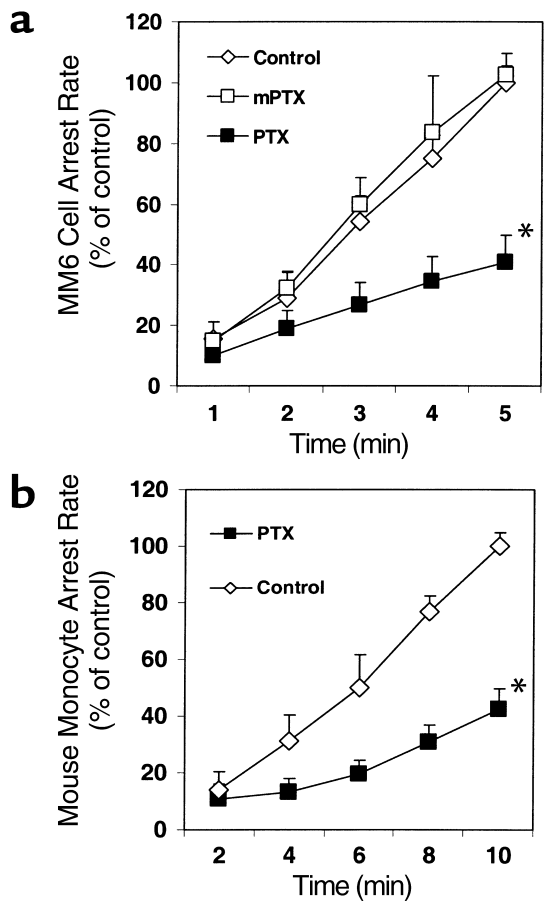

\section{Figure 1}

Monocyte activation through $\mathrm{G}_{i} \alpha$-coupled receptors promotes monocyte accumulation on native early atherosclerotic endothelium. (a) MM6 cell arrest in perfused apo $\mathrm{E}^{-/-}$mouse carotid arteries is significantly inhibited by pertussis toxin (PTX; ${ }^{*} P<0.01$, mean \pm SEM of six independent experiments per group), but not by mutant PTX (mPTX). (b) PTX inhibits mouse blood monocyte arrest on native early atherosclerotic endothelium $\left({ }^{*} P<0.01\right.$, mean \pm SEM of three independent experiments per group). 
a

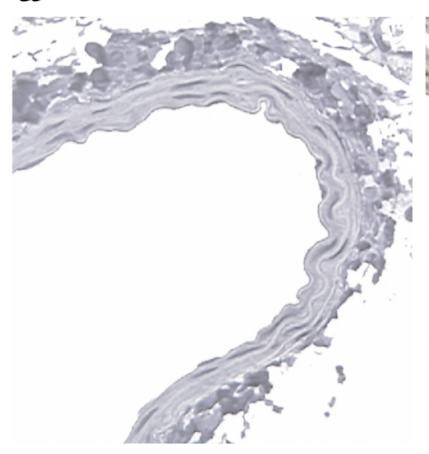

b

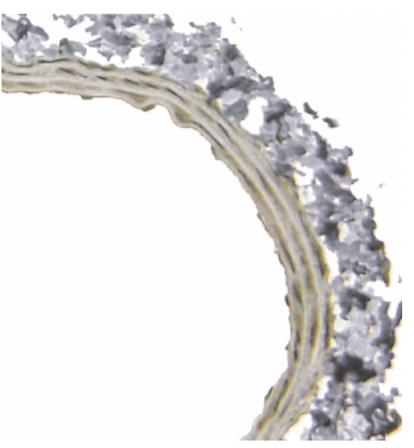

c

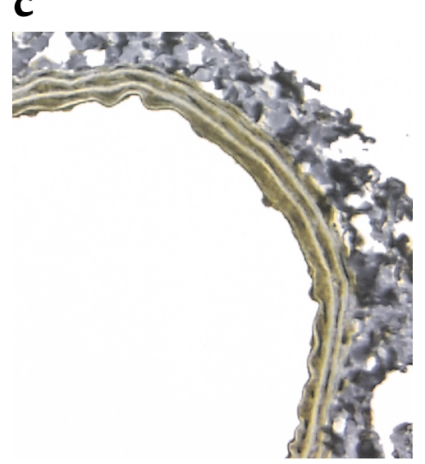

Figure 2

Chemokines expressed on early atherosclerotic endothelium of mouse carotid arteries. KC is strongly expressed on the endothelium and throughout the media of carotid arteries of apoE $\mathrm{E}^{-/-}$mice fed a Western diet for 5 weeks (b). JE (mouse MCP-1) is also highly expressed (c). Goat IgG produces no staining of the same vessel. (a). transmigration toward a gradient of murine JE in a Transwell-filter assay with a bell-shaped dose-dependence, and JE-induced migration was almost completely inhibited in the presence of a blocking JE $\mathrm{mAb}$ or the CCR2 peptide antagonist 9-76MCP-1 (Figure 3b). MM6 cells or mouse monocytes were treated with either 8-73GRO- $\alpha$ to block CXCR2, or 9-76MCP-1 to block CCR2, before perfusion through carotid arteries. The blocking effects of these antagonists have been demonstrated in both the human (25) and the murine systems (36). In this study, an effective blocking concentration of $5 \mu \mathrm{g} / \mathrm{ml}$ was used at which both 8-76GRO- $\alpha$ and 9-73MCP-1 were able to completely block an increase in intracellular calcium in MM6 cells and MM6 cell transmigration in response to the respective chemokine agonists (Figure $3 \mathrm{~b}$ and data not shown). Both receptor antagonists had no effect on MM6 cell or mouse monocyte tethering and rolling on the atherosclerotic endothelium. However, blockade of CXCR2 by 8-73GRO- $\alpha$ reduced MM6 cell accumulation in carotid arteries by $53 \%(n=6 ; P<0.01$; Figure $3 a)$ and mouse monocyte accumulation by $47 \%(n=5 ; P<0.01$; Figure $3 c$ ) indicating that CXCR2 was responsible for mediating arrest of rolling monocytes. By contrast, blocking CCR-2 by $9-76 \mathrm{MCP}-1$ or neutralizing JE on the carotid endothelium with an antibody had no effect on both MM6 arrest $(n=6$; $P=$ NS; Figure $3 \mathrm{a})$ or mouse monocyte arrest $(n=3 ; P=\mathrm{NS} ;$ Figure $3 \mathrm{c})$. To evaluate whether some of the MM6 cell accumulation blocked by 8-73GRO- $\alpha$ was mediated through G proteins other than $G_{i} \alpha$, we incubated MM6 cells with both PTX and $8-73$ GRO- $\alpha$. This caused no further reduction beyond the extent of inhibition seen with PTX alone $(n=4$; $P=$ NS; Figure 4).

To assess which chemokines are responsible for CXCR2-mediated monocyte arrest, monocyte suspensions were perfused after pretreatment of carotid arteries with blocking antibodies to the CXCR2 ligands KC or MIP-2, a chemokine that can also bind and activate CXCR2 (26). MM6 cell arrest was decreased by $41 \%$ with the KC antibody, whereas the MIP-2 antibody had no effect $(n=6$; $P<0.01$; Figure 4). Blocking MIP-2 in addition to blocking $\mathrm{KC}$ had no further effect ( $n=4 ; P=\mathrm{NS}$; Figure 4). Combining pretreatment of monocyte with the CXCR2 inhibitor 8-73GRO- $\alpha$ and pretreatment of the vessel with KC antibody provided no further inhibition of MM6 cell arrest beyond the effect of CXCR 2 inhibitor $(n=6$; $P=\mathrm{NS}$; Figure 4). This suggests that KC accounts for all MM6 cell arrest mediated by CXCR2. a

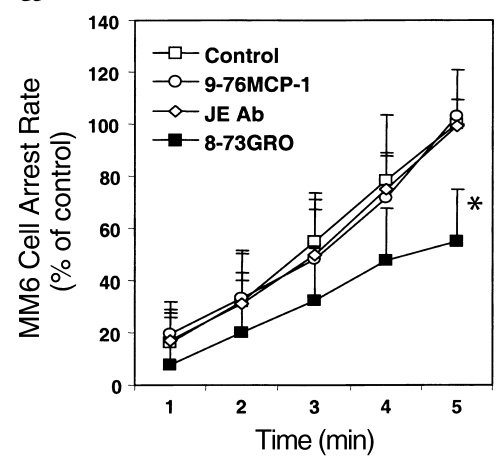

b

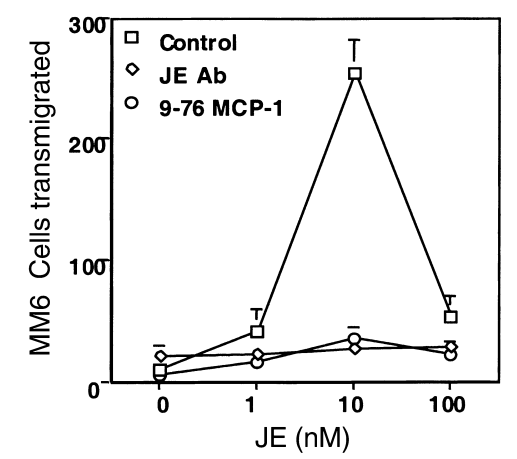

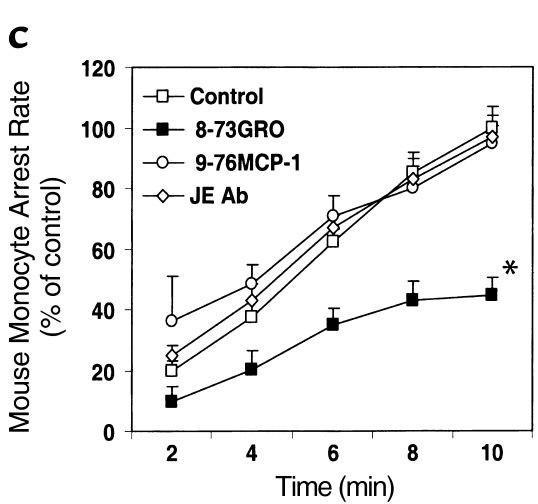

Figure 3

Effects of blocking chemokines and chemokine receptors on MM6 cell arrest on early atherosclerotic endothelium. (a) Blockade of CXCR2 by 8-73GRO, but neither blockade of CCR2 by 9-76MCP-1 nor neutralization of JE by a blocking antibody, leads to a decrease in MM6 cell arrest ( ${ }^{*} P<0.01$, mean $\left.\pm \mathrm{SEM}, n=6\right)$. (b) The $9-76 \mathrm{MCP}-1(5 \mu \mathrm{g} / \mathrm{ml})$ and JE blocking antibody $(10 \mu \mathrm{g} / \mathrm{ml})$ each completely inhibit MM6 cell transmigration response to JE (three experiments). (c) Blockade of CXCR2 by 8-73GRO but not CCR2 by 9-76MCP-1 or of JE by a blocking antibody leads to decreased mouse monocyte arrest $\left({ }^{*} P<0.01\right.$, mean $\left.\pm \mathrm{SEM}, n=5\right)$. 


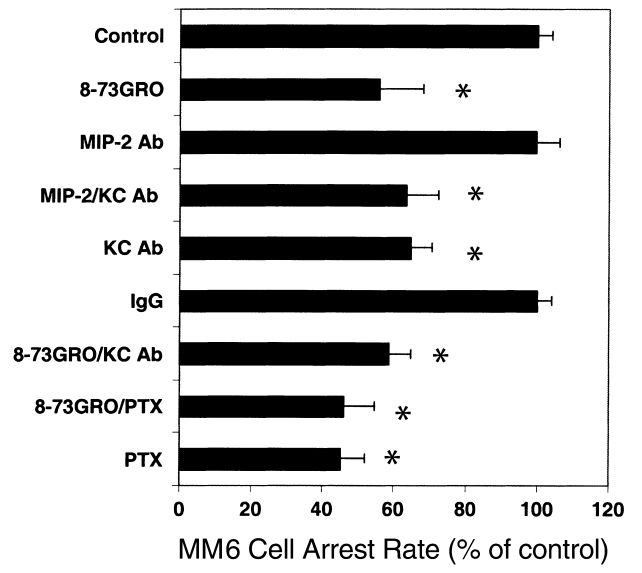

Figure 4

Role of KC on MM6 cell arrest on early atherosclerotic endothelium. MM6 cell accumulation at 5 minutes, normalized to control. Blocking CXCR2 with 8-73GRO, KC with an antibody, or both reduces cell accumulation ( ${ }^{*} P<0.01$, mean $\pm \mathrm{SEM}, n=6$ ). An MIP-2 antibody has no effect, either alone or in combination with KC antibody. Blocking CXCR2 with 8-73GRO does not further reduce accumulation of PTX-treated cells.

We have previously shown that perfusing the chemokine RANTES through mouse carotid arteries can increase the accumulation of MM6 cells (38). To test whether KC or MCP-1 has the same effect, we perfused carotid arteries from apoE- $/$ - mice with $\mathrm{KC}$ or MCP-1 at $100 \mathrm{nM}$ for 5 minutes. The number of arrested MM6 cells or mouse monocytes on the early atherosclerotic endothelium was more than doubled after KC perfusion, but perfusion of JE had no effect $(n=4 ; P<0.01$; Figure 5a; $n=3 ; P<0.01$; Figure $5 \mathrm{~b}$ ).

Monocyte arrest triggered by KC is mediated by VLA-4. To test which integrins were involved in chemokine-mediated monocyte arrest on atherosclerotic endothelium, we blocked VLA-4 or CD18 integrin on MM6 cells. Blockade of VLA-4 ( $n=4$; $P<0.01$; Figure $6 a)$ but not CD18 dramatically inhibited MM6 cell arrest on early atherosclerotic endothelium. VLA-4 blockade also abrogated the increase MM 6 cell arrest after $\mathrm{KC}$ perfusion, whereas antibody against CD18 did not $(n=4 ; P<0.01$; Figure $6 \mathrm{~b})$.

Similar results were obtained by perfusing mouse monocytes through carotid arteries. mAb PS/2 against $\alpha_{4}$ almost completely blocked monocyte accumulation $(n=4 ; P<0.01$; Figure $6 c)$, but mAb GAME- 46 to $\beta_{2}$ integrins had no effect. Blocking the VLA-4 ligand VCAM-1 with mAb M/K-2 but not the CD18 ligand ICAM-1 with mAb YN1 dramatically reduced mouse monocyte accumulation $(n=4 ; P<0.01$; Figure $6 c)$. This indicates that monocyte arrest triggered by $\mathrm{KC}$ via CXCR2 is mediated by activation of VLA- 4 .

\section{Discussion}

We demonstrate here that the chemokines $\mathrm{KC}$ and MCP-1 are expressed on native atherosclerotic endothelium but that only KC significantly contributes to monocyte arrest. Chemokines are found on the luminal surface and also diffusely distributed throughout the vessel wall even during early atherosclerosis. Although effects of chemokines on leukocyte adhesion, migration, and differentiation have been intensively studied, the role of endothelial-associated chemokines in leukocyte arrest has only recently received interest. This ex vivo perfused carotid artery model for the first time to our knowledge allows direct investigation of the role of chemokines in monocyte arrest on native early atherosclerotic endothelium.

Surprisingly, MCP-1, although expressed on the early atherosclerotic endothelium, failed to affect monocyte arrest. MCP-1 and its ligand CCR2 have been shown to be involved in atherosclerosis. MCP-1 is expressed in vascular cells of atherosclerotic vessels throughout the disease (39). Mutant mice lacking MCP-1 or its receptor CCR2 develop smaller atherosclerotic lesions than control mice do $(22,23)$. Based on our study, it appears that this effect is not due to reduced arrest of monocyte on lesion-prone arteries. This contrasts with data obtained by Gerszten et al. (24), which showed that in a reconstituted system, preincubation with MCP-1 for 1 minute promoted monocyte adhesion to cultured endothelial cells expressing E-selectin and CD18 ligands. There are several relevant differences between monocyte arrest in the flow chamber assay (24) and in an atherosclerotic artery. First, the contact time between a rolling monocyte and the atherosclerotic endothelium is likely to be much shorter than 1 minute. At typical rolling velocities observed in large arteries (10), cells would roll

\section{Figure 5}

Additional loading of KC increases monocyte arrest on early atherosclerotic endothelium. (a) KC, but not JE, perfusion increases MM6 cell arrest on early atherosclerotic endothelium ( ${ }^{*} P<0.01$, mean \pm SEM, $n=3$ ). Increased accumulation is blocked by PTX. (b) KC, but not JE, perfusion increases mouse monocyte arrest on early atherosclerotic endothelium ( ${ }^{*} P<0.01$, mean $\pm \mathrm{SEM}, n=3$ ).
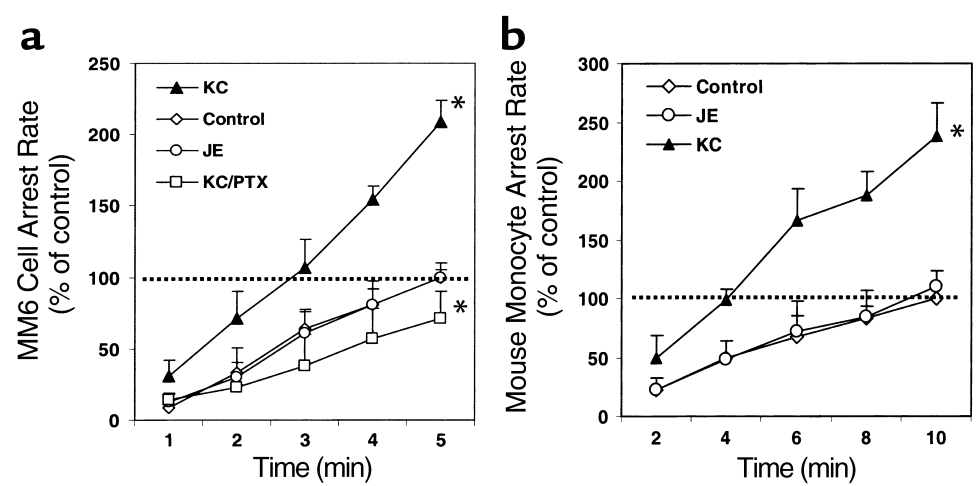

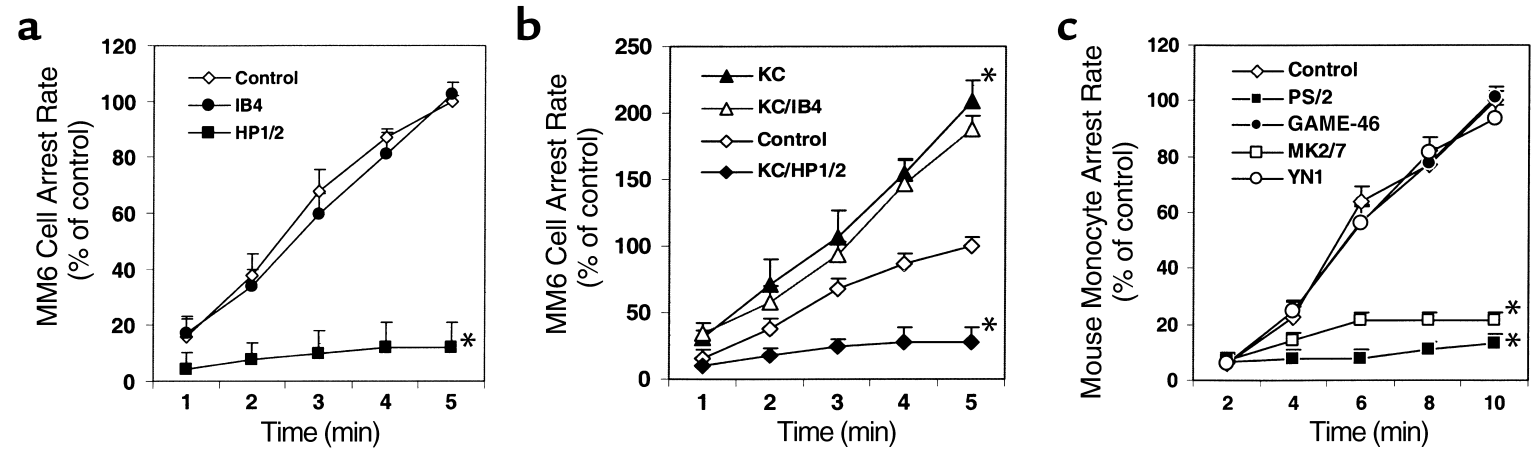

\section{Figure 6}

VLA-4 is responsible for chemokine-mediated MM6 arrest on early atherosclerotic endothelium. (a) Blocking VLA-4 (mAb HP1/2), but not CD18 (mAb IB4), blocks MM6 cell arrest on early atherosclerotic endothelium ( ${ }^{*} P<0.01$, mean $\left.\pm \mathrm{SEM}, n=3\right)$. (b) Increased MM6 cell arrest after KC perfusion is also inhibited by mAb HP1/2, but not IB4 (*P<0.01, mean $\pm \mathrm{SEM}, n=3)$. (c) Blocking VLA-4 (mAb PS/2) or VCAM-1 (mAb MK2/7), but not CD18 (mAb GAME-46) or ICAM-1 (mAb YN1), inhibits mouse blood monocyte arrest on early atherosclerotic endothelium $\left({ }^{*} P<0.01\right.$, mean $\left.\pm \mathrm{SEM}, n=3\right)$.

through a $1 \mathrm{~mm}$ long lesion-prone area in under 20 seconds. Although MCP-1 preincubation of monocytes in suspension can rapidly activate VLA-4 adhesiveness for VCAM-1 (40), it is possible that this activation of VLA-4 via CCR2 occurs with insufficient rapidity in shear flow. Next, in a rolling monocyte, only a few receptors contact the endothelium where the chemokine is presented. This is very different from incubation with soluble chemokine, where presumably all available receptors are triggered at the same time. In addition, it is possible that binding of MCP-1 to specific heparan sulfate proteoglycans may determine its presentation and immobilization on the endothelial surface and thereby its involvement in subsequent transmigration rather than initial arrest of monocytes (25). The present data reveal that MCP-1 and CCR2 do not contribute to triggering of monocyte arrest, suggesting that possibly MCP-1 and CCR2 are involved in the development of atherosclerosis by participating in subsequent monocyte transmigration, differentiation, or survival in the vessel wall.

Consistent with the role of GRO- $\alpha$ in monocyte arrest on cultured endothelial cells stimulated by TNF (25) or MM-LDL (41), we found that KC can trigger monocyte arrest in an atherosclerotic artery. The discrepancy in the ability of MCP-1 or KC to trigger monocyte arrest suggests differences in the signal transduction pathway or speed of integrin activation mediated by CCR 2 and CXCR2. Although no studies exist addressing this issue directly, it has been shown that the avidity of the $\beta_{1}$ integrins VLA- 4 and VLA-5, as well as $\beta_{2}$ integrins LFA- 1 and Mac- 1 , can differently and sequentially be upregulated via specific signaling pathways after mononuclear cells exposure to chemokines. Notably, it has been described that the induction of VLA-4 adhesiveness appeared to differ between different CC chemokines (40).

When we preperfused the carotid arteries with $\mathrm{KC}$, this caused an increased rate of monocyte accumulation. This finding suggests that the endothelial bind- ing sites for $\mathrm{KC}$ are not saturated on atherosclerotic endothelium in vivo. Furthermore, this observation shows that monocyte activation by KC presented on the endothelium is not maximal, but can be increased further. Taken together, these data suggest that $\mathrm{KC}$ is a critical and limiting factor in monocyte arrest.

$\mathrm{KC}$ is also a potent neutrophil chemoattractant (42, 43). Conditions for neutrophil arrest (selectins, chemokines, and integrin ligands) are available on early atherosclerotic endothelium. This suggests that KC may also arrest neutrophils on early atherosclerotic endothelium. However, neutrophils are rarely observed in atherosclerotic lesions (44). The reasons for this are not known but could reside in an inability to establish firm adhesion, migration, or infiltration of atherosclerotic lesions, e.g., owing to the requirement of VLA-4, which is less abundant on neutrophils than on monocytes.

In our ex vivo model, VLA-4 is responsible for nearly all chemokine-mediated monocyte arrest on early atherosclerotic endothelium. This finding was surprising, as chemokines have been shown to arrest rolling lymphocytes on adhesion molecule-coated surfaces or cultured endothelium via activation of VLA-4 (45) or LFA-1 $(17,24)$. The reason why VLA-4 but not CD18 integrins mediate monocyte arrest on early atherosclerotic endothelium is unclear. In contrast to LFA-1 or Mac-1, $\alpha_{4}$ integrins are predominately expressed on leukocyte microvilli (46), preferential sites of leukocyteendothelial contacts under shear flow. It is possible that the proximity between chemokine receptors and $\alpha_{4}$ integrin at these surface projections may facilitate the rapid coupling of chemokine receptor signal to modulation of $\alpha_{4}$ integrin activation at adhesive contact sites under shear flow. Recently, it has been suggested that VLA-4 can be activated extremely rapidly while the cell is tethering (47). It is also likely that rolling monocytes, contacting a variety of chemokines including KC and other chemokines present on early atherosclerotic endothelium, might also be able to acti- 
vate CD18 integrins. Indeed, mice lacking CD18 integrin (48) or one of their endothelial ligands, ICAM-1, are partially protected from atherosclerosis. However, based on our data, it appears that activated $\beta_{2}$ integrins may be involved in downstream events in monocyte recruitment in atherosclerosis, like stabilization of adhesion, transmigration, or differentiation, rather than in monocyte arrest.

In conclusion, our study identifies $\mathrm{KC}$ as the first chemokine responsible for monocyte arrest on native atherosclerotic endothelium. In isolated perfused carotid arteries of apoE-/- mice, about one half of the monocyte accumulation is $\mathrm{KC}$ dependent. Blocking KC by an antibody has about the same effect as blocking the $\mathrm{KC}$ receptor, CXCR2. The dominant role played by the chemokine KC and the adhesion molecules VLA-4 and VCAM-1 suggests that these molecules are interesting targets for therapeutic or preventive interventions in atherosclerosis.

\section{Acknowledgments}

We thank John Sanders for immunostaining, Michele Kirkpatrick for mouse husbandry, Erik Hewlett for PTX and MPTX advice, and I. Clark-Lewis at the University of British Columbia for the peptide antagonists. This work was supported by NIH grant HL-58108 to K. Ley, Deutsche Forschungsgemeinschaft grant We1913/2 to C. Weber, and American Heart Association Post-doctoral Fellowship award 0120404 U to Y. Huo.

1. Ross, R. 1999. Atherosclerosis is an inflammatory disease. Am. Heart J. 138:S419-S420.

2. Ross, R. 1995. Cell biology of atherosclerosis. Annu. Rev. Physiol. 57:791-804.

3. Gerrity, R.G. 1981. The role of the monocyte in atherogenesis. I. Transition of blood-borne monocytes into foam cells in fatty lesions. Am. J. Pathol. 103:181-190.

4. Gerrity, R.G. 1981. The role of the monocyte in atherogenesis. II. Migration of foam cells from atherosclerotic lesions. Am. J. Pathol. 103:191-200.

5. Johnson-Tidey, R.R., McGregor, J.L., Taylor, P.R., and Poston, R.N. 1994. Increase in the adhesion molecule P-selectin in endothelium overlying atherosclerotic plaques: coexpression with intercellular adhesion molecule-1. Am. J. Pathol. 144:952-961.

6. Iiyama, K., et al. 1999. Patterns of vascular cell adhesion molecule- 1 and intercellular adhesion molecule-1 expression in rabbit and mouse atherosclerotic lesions and at sites predisposed to lesion formation. Circ. Res. 85:199-207.

7. Sakai, A., et al. 1997. P-selectin and vascular cell adhesion molecule-1 are focally expressed in aortas of hypercholesterolemic rabbits before intimal accumulation of macrophages and T lymphocytes. Arterioscler. Thromb. Vasc. Biol. 17:310-316.

8. Nakashima, Y., Raines, E.W., Plump, A.S., Breslow, J.L., and Ross, R. 1998. Upregulation of VCAM-1 and ICAM-1 at atherosclerosis-prone sites on the endothelium in the apoE-deficient mouse. Arterioscler. Thromb. Vasc. Biol. 18:842-851.

9. Nakashima, Y., Plump, A.S., Raines, E.W., Breslow, J.L., and Ross, R. 1994. ApoE-deficient mice develop lesions of all phases of atherosclerosis throughout the arterial tree. Arterioscler. Thromb. 14:133-140.

10. Ramos, C.L., et al. 1999. Direct demonstration of P-selectin- and VCAM1-dependent mononuclear cell rolling in early atherosclerotic lesions of apolipoprotein E-deficient mice. Circ. Res. 84:1237-1244.

11. Huo, Y., Hafezi-Moghadam, A., and Ley, K. 2000. Role of vascular cell adhesion molecule- 1 and fibronectin connecting segment- 1 in monocyte rolling and adhesion on early atherosclerotic lesions. Circ. Res. 87:153-159.

12. Dong, Z.M., Brown, A.A., and Wagner, D.D. 2000. Prominent role of Pselectin in the development of advanced atherosclerosis in ApoE-deficient mice. Circulation. 101:2290-2295.

13. Collins, R.G., et al. 2000. P-selectin or intercellular adhesion molecule
(ICAM)-1 deficiency substantially protects against atherosclerosis in apolipoprotein E-deficient mice. J. Exp. Med. 191:189-194.

14. Shih, P.T., et al. 1999. Blocking very late antigen-4 integrin decreases leukocyte entry and fatty streak formation in mice fed an atherogenic diet. Circ. Res. 84:345-351.

15. Haribabu, B., et al. 1999. Chemoattractant receptors activate distinct pathways for chemotaxis and secretion. Role of G-protein usage. J. Biol. Chem. 274:37087-37092.

16. Murdoch, C., and Finn, A. 2000. Chemokine receptors and their role in inflammation and infectious diseases. Blood. 95:3032-3043.

17. Campbell, J.J., et al. 1998. Chemokines and the arrest of lymphocytes rolling under flow conditions. Science. 279:381-384.

18. Takeya, M., Yoshimura, T., Leonard, E.J., and Takahashi, K. 1993. Detection of monocyte chemoattractant protein-1 in human atherosclerotic lesions by an anti-monocyte chemoattractant protein- 1 monoclonal antibody. Hum. Pathol. 24:534-539.

19. Koch, A.E., et al. 1993. Enhanced production of the chemotactic cytokines interleukin- 8 and monocyte chemoattractant protein- 1 in human abdominal aortic aneurysms. Am. J. Pathol. 142:1423-1431.

20. Pattison, J.M., Nelson, P.J., Huie, P., Sibley, R.K., and Krensky, A.M. 1996. RANTES chemokine expression in transplant-associated accelerated atherosclerosis. J. Heart Lung Transplant. 15:1194-1199.

21. Wilcox, J.N., Nelken, N.A., Coughlin, S.R., Gordon, D., and Schall, T.J. 1994. Local expression of inflammatory cytokines in human atherosclerotic plaques. J. Atheroscler. Thromb. 1(Suppl. 1):S10-S13.

22. Gu, L., et al. 1998. Absence of monocyte chemoattractant protein-1 reduces atherosclerosis in low density lipoprotein receptor-deficient mice. Mol. Cell. 2:275-281.

23. Boring, L., Gosling, J., Cleary, M., and Charo, I.F. 1998. Decreased lesion formation in CCR2 $(-/-)$ mice reveals a role for chemokines in the initiation of atherosclerosis. Nature. 394:894-897.

24. Gerszten, R.E., et al. 1999. MCP-1 and IL-8 trigger firm adhesion of monocytes to vascular endothelium under flow conditions. Nature. 398:718-723

25. Weber, K.S.C., von Hundelshausen, P., Clark-Lewis, I., Weber, P.C., and Weber, C. 1999. Differential immobilization and hierarchical involvement of chemokines in monocyte arrest and transmigration on inflamed endothelium in shear flow. Eur. J. Immunol. 29:700-712.

26. Bozic, C.R., et al. 1994. The murine interleukin 8 type B receptor homologue and its ligands. Expression and biological characterization. J. Biol. Chem. 269:29355-29358.

27. Yoshimura, T., et al. 1989. Human monocyte chemoattractant protein1 (MCP-1). Full-length cDNA cloning, expression in mitogen-stimulated blood mononuclear leukocytes, and sequence similarity to mouse competence gene JE. FEBS Lett. 244:487-493.

28. Blackmore, P.F., Bocckino, S.B., Waynick, L.E., and Exton, J.H. 1985. Role of a guanine nucleotide-binding regulatory protein in the hydrolysis of hepatocyte phosphatidylinositol 4,5-bisphosphate by calcium-mobilizing hormones and the control of cell calcium. Studies utilizing aluminum fluoride. J. Biol. Chem. 260:14477-14483.

29. Pizza, M., et al. 1989. Mutants of pertussis toxin suitable for vaccine development. Science. 246:497-500.

30. Jones, S.A., Dewald, B., Clark-Lewis, I., and Baggiolini, M. 1997. Chemokine antagonists that discriminate between interleukin- 8 receptors. Selective blockers of CXCR2. J. Biol. Chem. 272:16166-16169.

31. Gong, J.H., and Clark-Lewis, I. 1995. Antagonists of monocyte chemoattractant protein 1 identified by modification of functionally critical NH2-terminal residues. J. Exp. Med. 181:631-640.

32. Zhang, Y.J., Rutledge, B.J., and Rollins, B.J. 1994. Structure/activity analysis of human monocyte chemoattractant protein-1 (MCP-1) by mutagenesis. Identification of a mutated protein that inhibits MCP-1-mediated monocyte chemotaxis. J. Biol. Chem. 269:15918-15924.

33. Ziegler-Heitbrock, H.W., et al. 1988. Establishment of a human cell line (Mono Mac 6) with characteristics of mature monocytes. Int. J. Cancer. 41:456-461.

34. Jung, S., et al. 2000. Analysis of fractalkine receptor CX(3)CR1 function by targeted deletion and green fluorescent protein reporter gene insertion. Mol. Cell. Biol. 20:4106-4114.

35. Jung, U., and Ley, K. 1999. Mice lacking two or all three selectins demonstrate overlapping and distinct functions of each selectin. J. Immunol. 162:6755-6762.

36. Gong, J.H., Ratkay, L.G., Waterfield, J.D., and Clark-Lewis, I. 1997. An antagonist of monocyte chemoattractant protein 1 (MCP-1) inhibits arthritis in the MRL-lpr mouse model. J. Exp. Med. 186:131-137.

37. Weber, C., Lu, C.F., Casasnovas, J.M., and Springer, T.A. 1997. Role of alpha 1 beta 2 integrin avidity in transendothelial chemotaxis of mononuclear cells. J. Immunol. 159:3968-3975.

38. von Hundelshausen, P., et al. 2001. Rantes deposition by platelets triggers monocyte arrest on inflamed and atherosclerotic endothelium. Circulation. 103:1772-1777.

39. Reape, T.J., and Groot, P.H. 1999. Chemokines and atherosclerosis. Ath- 
erosclerosis. 147:213-225.

40. Weber, C., Alon, R., Moser, B., and Springer, T.A. 1996. Sequential regulation of alpha 4 beta 1 and alpha 5 beta 1 integrin avidity by $\mathrm{cc}$ chemokines in monocytes: implications for transendothelial chemotaxis. J. Cell Biol. 134:1063-1073.

41. Schwartz, D., et al. 1994. Role of the GRO family of chemokines in monocyte adhesion to MM-LDL-stimulated endothelium. J. Clin. Invest. 94:1968-1973.

42. Ahuja, S.K., and Murphy, P.M. 1996. The CXC chemokines growth-regulated oncogene (GRO) alpha, GRObeta, GROgamma, neutrophil-activating peptide- 2 , and epithelial cell-derived neutrophil-activating peptide-78 are potent agonists for the type $\mathrm{B}$, but not the type $\mathrm{A}$, human interleukin-8 receptor. J. Biol. Chem. 271:20545-20550.

43. Hoch, R.C., Schraufstatter, I.U., and Cochrane, C.G. 1996. In vivo, in vitro, and molecular aspects of interleukin- 8 and the interleukin-8 receptors. J. Lab. Clin. Med. 128:134-145.
44. Ross, R. 1993. The pathogenesis of atherosclerosis: a perspective for the 1990s. Nature. 362:801-809.

45. Honda, S., et al. 1994. Ligand-induced adhesion to activated endothelium and to vascular cell adhesion molecule- 1 in lymphocytes transfected with the $\mathrm{N}$-formyl peptide receptor. J. Immunol. 152:4026-4035.

46. Abitorabi, M.A., Pachynski, R.K., Ferrando, R.E., Tidswell, M., and Erle, D.J. 1997. Presentation of integrins on leukocyte microvilli: a role for the extracellular domain in determining membrane localization. J. Cell Biol. 139:563-571.

47. Grabovsky, V., et al. 2000. Subsecond induction of alpha4 integrin clustering by immobilized chemokines stimulates leukocyte tethering and rolling on endothelial vascular cell adhesion molecule 1 under flow conditions. J. Exp. Med. 192:495-506.

48. Nageh, M.F., et al. 1997. Deficiency of inflammatory cell adhesion molecules protects against atherosclerosis in mice. Arterioscler. Thromb. Vasc. Biol. 17:1517-1520. 